\title{
Bedeutung der Genetik in der klinischen Medizin
}

\author{
Andreas Huber ${ }^{a}$, Reto Stocker ${ }^{b}$, Thomas Szucs ${ }^{c}$ \\ a Prof. Dr. med., Kantonsspital Aarau, Institut für Labormedizin; b Prof. Dr. med., Klinik Hirslanden Zürich, Institut für Anästhesiologie und Intensivmedizin; \\ c Prof. Dr. med., Universität Basel, European Center of Pharmaceutical Medicine
}

Hinweis

Lesen Sie zu diesem Thema

auch den nachfolgenden

der genetischen und

genomischen Medizin

in der Schweiz» von

der Schweizerischen

Gesellschaft für

Medizinische Genetik

(SGMG)
Beitrag «Entwicklung

Die Genetik macht rasante Fortschritte und reicht mittlerweile tief in den klinischen Alltag hinein. In der täglichen Praxis sind die verschiedenen Stakeholder im Gesundheitswesen zu wenig für diese anspruchsvolle Herausforderung vorbereitet. Um das Verständnis zu fördern und eine adäquate Diagnose, Therapie und vor allem Beratung für alle Patientinnen und Patienten zu gewährleisten, sind auf allen Ebenen, nämlich Aus-, Weiter- und Fortbildung, rasch Massnahmen zu ergreifen, welche das Know-how zu klinischer Genomik und Genetic Counselling fördern. Dabei sind alle Lehrbetriebe und alle Fachgesellschaften wie auch private und öffentliche Institutionen zu beteiligen.

\section{Ausgangslage}

Mit der Entschlüsselung des humanen Genoms und vor allem mit der Realisierung von neuen diagnostischen Methoden wie Next-Generation-Sequencing (NGS) hat die Genetik rasch einen grossen Einfluss auf die klinische Medizin bekommen. Heute sind schon Panels zur Verfügung, mit denen relativ einfach und rasch mehrere hundert Gene analysiert werden können. Man findet fast täglich neue wissenschaftliche Literatur zur Genetik. Viele schon lange bekannte vererbbare Krankheiten und Syndrome sind heute molekulargenetisch auf das Genauste definiert, ver-

\section{Résumé}

La génétique progresse rapidement et ses ramifications s'étendent maintenant profondément dans le quotidien clinique. Dans la pratique, les différents acteurs du système de santé ne sont pas suffisamment préparés pour relever ce défi ambitieux. Afin de promouvoir la compréhension et d'assurer un diagnostic, une thérapie et, surtout, des conseils adéquats à l'ensemble des patients, des mesures doivent être prises rapidement à tous les niveaux, à savoir la formation initiale, postgraduée et continue pour améliorer les connaissances en termes de génomique clinique et de conseil génétique. Tous les établissements formateurs et associations professionnelles de même que les institutions privées et publiques doivent être impliqués dans ce cadre. schiedene Varianten bekannt, deren Bedeutung charakterisiert. Allerdings sind die Erbgänge nicht immer nur einfach nach den mendelschen Regeln, sondern oft komplizierter. Der Weg vom Genotyp zum Phänotyp hängt unter anderem auch von der Penetranz ab, weshalb Vorhersagen zu konkreten Auswirkung eines Gendefektes auf einen Patienten nicht so trivial sind. Als Beispiel der nicht ganz einfachen Interpretation von Mutationen sei das BRCA1/2-Gen genannt. Man kann hier Mutationen finden, die ganz klar mit einem erhöhten Brustkrebsrisiko vergesellschaftet sind. Es gibt aber auch Mutationen oder Varianten, die sicher nicht mit einem erhöhten Brustkrebsrisiko vergesellschaftet sind, und schliesslich gibt es sogenannte variances of unknown significance (VUS). Bei diesen ist die Datenlage nicht sicher, womit auch die Beratung einer betroffenen Patientin und die Entscheidung zur diagnostischen oder therapeutischen Massnahme schwierig wird. Zunehmend wird Gendiagnostik auch eingesetzt zur Abschätzung der Verstoffwechselung eines Medikamentes bei einem einzelnen Individuum. So nimmt die Pharmakogenomik langsam, aber Schritt für Schritt, Einfluss in die klinische Medizin resp. die Pharmakologie.

Auch in der Nicht-Keimbahn Genetik also bei erworbenen DNA / Gendefekten hat die moderne molekularbiologische und zytogenetische Diagnostik viele Erkenntnisse geliefert. Vor allem bei malignen Tumoren 
sind genetische Veränderungen wichtig für Diagnose, Prognose und Prädiktion von Ansprechen auf Therapie. Heute schon und sicher noch viel mehr in der Zukunft beeinflusst das genetische Make-up eines Tumors die Auswahl der Therapie, z.B. die Wahl eines spezifischen Chemotherapeutikums.

\section{Problemstellung}

Zweifelsohne ist es ausgesprochen schwierig diese rasche Entwicklung in die medizinischen Aus-, Weiterund Fortbildungscurricula aufzunehmen. Gut wurden die entsprechenden Themen aus der Genetik im Medizinstudium auf verschiedenen Ebenen in den verschiedenen Fächern integriert und werden heute entsprechend gelehrt. Auch haben die Fachgesellschaften ihrerseits diese Gen-Themen teilweise in ihre Weiterbildungscurricula einfliessen lassen. Und man kann davon ausgehen, dass diese Themen auch bei der praktischen Weiterbildung im Spital, in der Klinik und in der Praxis entsprechend gewichtet vorkommen. Sicher bestehen jedoch grosse Unterschiede. Es gibt den Weiterbildungsgang zum Facharzt für «Medizinische Genetik» und für "Medizinisch-genetische Analytik FAMH», wodurch der Nachwuchs an genetischen Fachkräften sichergestellt werden soll. Allerdings ist die Zahl der Titelträger «Medizinische Genetik und «Medizinisch-genetische Analytik FAMH» und auch die Zahl der in Weiterbildung befindlichen Personen in der Schweiz ungenügend. Der Bedarf an solchen Fachkräften ist grösser als was die Weiterbildung derzeit generieren kann. Ebenso fehlt ein Lehrgang zu einem Fähigkeitsausweis "Medizinische Genetik» oder gar ein entsprechender Schwerpunkt-Titel. Es sind Bestrebungen der Gesellschaft für medizinische Genetik SGMG und der SAMW im Gange, diese Aus, Weiter- und Fortbildungsdefizite zu analysieren. Letztlich sind auch alle seit längerem in der Praxis oder im Spital tätigen Ärztinnen und Ärzte gefordert, welche ihre Aus- und Weiterbildung zu einem Zeitpunkt abgeschlossen haben, als das genetische Wissen noch nicht so dominant war. Der praktisch tätige Arzt, die praktisch tätige Ärztin sind gehalten, ihr Wissen durch Besuch von Fortbildungsanlässen oder aber durch Literaturstudium aufzufrischen. Dazu gibt es eine ganz beschauliche Palette an Fortbildungsveranstaltungen, bei denen auch genetische Aspekte dazugekommen sind. Trotzdem scheint aber auch hier nur ein unzureichendes Angebot zu bestehen, um den täglichen klinischen Situationen gerecht zu werden.

Um den Mediziner zu entlasten und das genetische Knowhow zu vertiefen und vor allem an die Patienten heranzubringen, wurden in vielen Ländern Lehrgänge für sogenannte Genetic Counsellors etabliert. Diese Fachkräfte, sei deren Hintergrund Biologie oder Krankenpflege, werden von einem genetisch versierten Facharzt überwacht und eingesetzt. In der Schweiz gibt es keinen solchen Bildungsgang und infolge dessen auch keinen offiziellen Titel «Genetic Counsellor». In der Berufswelt finden sich ein paar wenige Personen, die ihre Weiterbildung im Ausland gemacht haben und nun in der Schweiz als Genetic Counsellors in grossen Spitälern arbeiten. Es dürfte aber noch eine Weile gehen, bis ein solcher neuer Lehrgang etabliert ist und genügend Fachkräfte weitergebildet sein werden. Deswegen bleibt im Moment nur übrig, entweder diese Fachkräfte aus dem Ausland zu rekrutieren oder aber schweizerische Fachleute im Ausland weiterbilden zu lassen, um sie dann wieder bei uns einzustellen.

\section{Lösungsansätze}

Es müssen Wege gefunden werden, auf der Ebene von Aus- und Weiterbildung, aber vor allem auch im Rahmen der Fortbildung Angebote zu etablieren, welche die Bildung der wichtigen Akteure im Gesundheitswesen in relevanten Genetik-Themen (z.B. Genom, genomische Variabilität, Untersuchungsmethoden, Bioinformatik, genetische Erkrankungen inkl. Behandlungsmöglichkeiten, Beratung von Patienten, ethische und legale Aspekte) erlauben. Dabei geht es um Bildung von klinisch Tätigen wie Ärzte, Pflegepersonal, Hebammen und Laborfachkräfte, damit die Kenntnisse dann in der klinischen Medizin vertieft Einzug finden.

In einem ersten CAS offeriert die Universität Fürstentum Liechtenstein (UFL) einen einjährigen berufsbegleitenden Lehrgang, in welchem die klinisch-genomische Medizin sehr umfassend abgehandelt wird. Des Weiteren sollten die verschiedenen medizinischen Fachgesellschaften zusammen mit dem schweizerischen Institut für Weiter- und Fortbildung (SIWF), Wege finden, wie ein Fähigkeitsausweis «Medizinische Genetik» etabliert werden kann. Weiter wäre zu überlegen, ob auch ein Schwerpunkt-Titel zusätzlich zum erworbenen Facharzt-Titel wie z.B. Hämatologie, Neurologie, Pädiatrie, Geburtshilfe usw. ergänzend entwickelt werden soll. So oder so sind die Fachgesellschaften, das SIWF, die FAMH wie auch Hochschulen und Vertreter der Weiterbildungsprogramme an Spitälern unter Mithilfe von BAG, allenfalls die Gesundheitsdirektoren-Konferenz und vielleicht auch die Bildungsdirektoren-Konferenz gefordert. 